\title{
A NOTE ON ISOMETRIC IMMERSIONS
}

\section{BAIKOUSSIS and F. BRICKELL.}

(Received 11 December 1980; revised 15 June 1981)

Communicated by K. Mackenzie

\begin{abstract}
Let $N$ be a complete connected Riemannian manifold with sectional curvatures bounded from below. Let $M$ be a complete simply connected Riemannian manifold with sectional curvatures $K_{M}(\sigma) \leqslant-a^{2}$ $(a \geqslant 0)$ and with dimension $<2 \operatorname{dim} N$. Suppose that $N$ is isometrically immersed in $M$ and that its image lies in a closed ball of radius $\rho$. Then $\sup \left(K_{N}(\sigma)-K_{M}(\sigma)\right) \geqslant \mu^{2}(a \rho) / \rho^{2}$ where the function $\mu$ is defined by $\mu(x)=x \operatorname{coth} x$ for $x>0, \mu(0)=1$ and the supremum is taken over all sections tangent to $N$.
\end{abstract}

1980 Mathematics subject classification (Amer. Math. Soc): 53 C 40.

This is a generalisation of previous results by Jacobowitz [4], Moore [6], Baikoussis and Koufogiorgos [1] and Ishihara [3]. To prove the main theorem we need the following

LEMMA 1. Let $M$ be a Riemannian manifold with sectional curvatures $\leqslant-a^{2}$. Suppose that $\gamma:[0,1] \rightarrow M$ is a geodesic and put $T=\gamma^{\prime}(t)$. Let $V$ be a Jacobi field along $\gamma$ which is zero to $t=0$ and is everywhere perpendicular to $\gamma$. Then at $t=1$.

$$
\frac{\left\langle\nabla_{T} V, V\right\rangle}{\langle V, V\rangle} \geqslant \mu(a \lambda)
$$

where $\lambda$ is the length of $\gamma$.

Proof. This can be extracted from the proof of the Rauch Comparison Theorem given in [2]. On page 32 of this reference the inequality

$$
\frac{\left\langle\nabla_{T} V, V\right\rangle}{\langle V, V\rangle} \geqslant \frac{\left\langle\nabla_{T} V_{0}, V_{0}\right\rangle}{\left\langle V_{0}, V_{0}\right\rangle}
$$

This work was done during the time when the first author was a visitor at the University of Southampton.

c. Copyright Australian Mathematical Society 1982 
occurs where the subscript zero refers to a comparison manifold $M_{0}$. Our lemma follows by choosing an appropriate Jacobi field in the complete simply connected space of constant sectional curvature $-a^{2}$.

The following algebraic lemma is proved on pages $28-29$ of [5].

Lemma 2. Let $S: R^{n} \times R^{n} \rightarrow R^{p}$ be a symmetric bilinear function such that, for all $X \neq 0, S(X, X) \neq 0$. Then if $p<n$ we can find non-zero vectors $X, Y$ such that $S(X, Y)=0, S(X, X)=S(Y, Y)$.

THEOREM. Let $N$ be a complete connected Riemannian manifold with sectional curvature bounded from below. Let $M$ be a complete simply connected Riemannian manifold with sectional curvatures $K_{M}(\sigma) \leqslant-a^{2}(a \geqslant 0)$ and with $\operatorname{dim} M<$ $2 \operatorname{dim} N$. Suppose that $N$ is isometrically immersed in $M$ and that its image lies in a closed ball of radius $\rho$. Then

$$
\sup \left(K_{N}(\sigma)-K_{M}(\sigma)\right) \geqslant \mu^{2}(a \rho) / \rho^{2} .
$$

Proof. In order to simplify the notation we shall assume that $N$ is imbedded in $M$. The Riemannian connections $\nabla$ and $\nabla^{\prime}$ of $M$ and $N$ respectively are related by the Gauss formula

$$
\nabla_{X} Y=\nabla_{X}^{\prime} Y+S(X, Y)
$$

where $S$ is the second fundamental form of the immersion. The corresponding sectional curvatures are related by the Gauss equation

$$
K_{N}(X \wedge Y)-K_{M}(X \wedge Y)=\Delta(X, Y)
$$

where

$$
\Delta(X, Y)=\frac{\langle S(X, X), S(Y, Y)\rangle\rangle-\|S(X, Y)\|^{2}}{\|X \wedge Y\|^{2}}
$$

Now let 0 be the centre of the ball in $M$ and consider the function $\Phi$ defined on $N$ by $\Phi(P)=\frac{1}{2}\{d(0, P)\}^{2}$ where $d$ is the distance function on $M$. Our theorem follows from an application of Theorem $A^{\prime}$ in [7] to the function $\Phi$ but we have first to do some calculations.

Consider a unit vector $X$ tangent to $N$ at $P$ and choose a curve $\beta(u)$ in $N$ with $\beta(0)=P, \beta^{\prime}(0)=X$. Let $a(t, u), 0 \leqslant t \leqslant 1$, be a constant speed parametrisation of the (unique) geodesic in $M$ from 0 to $\beta(u)$ and define vector fields $T, \tilde{X}$ along $a$ by

$$
T=a_{*} \frac{\partial}{\partial t}, \quad \tilde{X}=a_{*} \frac{\partial}{\partial u}
$$


we have the formulas

$$
\begin{gathered}
\langle\operatorname{grad} \Phi, X\rangle=\langle T, X\rangle \\
\nabla^{\prime 2} \Phi(X, X)=\left\langle\nabla_{T} \tilde{X}, X\right\rangle+\langle T, S(X, X)\rangle,
\end{gathered}
$$

where $\nabla^{\prime 2} \Phi$ is the Hessian of $\Phi$. The first of these is a straightforward calculation involving the first variation. The second one can be derived as follows. Put $\bar{X}=\tilde{X}(1, u)$ so that $\bar{X}$ is a vector field along $\beta$ which is tangent to $N$. Then

$$
\nabla^{\prime 2} \Phi(X, X)=\left\langle\nabla_{X}^{\prime} \operatorname{grad} \Phi, X\right\rangle=X(\langle\operatorname{grad} \Phi, \bar{X}\rangle)-\left\langle\operatorname{grad} \Phi, \nabla_{X}^{\prime} \bar{X}\right\rangle .
$$

Now use (5), (2) and the fact that, because $[T, \tilde{X}]=0, \nabla_{\tilde{X}} T=\nabla_{T} \tilde{X}$ to obtain

$$
\begin{aligned}
\nabla^{\prime 2} \Phi(X, X) & =X(\langle T, \tilde{X}\rangle)-\left\langle T, \nabla_{X}^{\prime} \bar{X}\right\rangle \\
& =\left\langle\nabla_{X} T, \bar{X}\right\rangle+\left\langle T, \nabla_{X} \bar{X}-\nabla_{X}^{\prime} \bar{X}\right\rangle \\
& =\left\langle\nabla_{T} \tilde{X}, X\right\rangle+\langle T, S(X, X)\rangle .
\end{aligned}
$$

For our next calculations we restrict the vector fields to the geodesic $\gamma: t \rightarrow$ $a(t, 0)$. Because $\tilde{X}$ is a Jacobi field it follows that

$$
T^{2}(\langle T, \tilde{X}\rangle)=0 \text { and consequently }\langle T, \tilde{X}\rangle=k t
$$

where $k=\langle T, X\rangle$. The vector field

$$
\hat{X}=\tilde{X}-\frac{\langle T, \tilde{X}\rangle T}{\lambda^{2}}=\tilde{X}-\frac{k t T}{\lambda^{2}},
$$

where $\lambda=\|T\|$ is the length of $\gamma$, is thus a Jacobi field which is everywhere perpendicular to $\gamma$. A calculation gives the relations

$$
\begin{gathered}
\langle\tilde{X}, \tilde{X}\rangle=\langle\hat{X}, \hat{X}\rangle+k^{2} t^{2} / \lambda^{2} \\
\left\langle\nabla_{T} \tilde{X}, \tilde{X}\right\rangle=\left\langle\nabla_{T} \hat{X}, \hat{X}\right\rangle+k^{2} t^{2} / \lambda^{2} .
\end{gathered}
$$

Choose a point $P_{0}$ on $N$ different from 0 and put $\lambda_{0}=d\left(0, P_{0}\right)$. According to Theorem $\mathrm{A}^{\prime}$ of [7], for any $\varepsilon^{\prime}>0, \varepsilon>0$, there exists a point $P$ on $N$ at which

$$
d(0, P) \geqslant \lambda_{0}, \quad\|\operatorname{grad} \Phi\|<\varepsilon^{\prime}, \quad \nabla^{\prime 2} \Phi(X, X)<\varepsilon,
$$

where $X$ is any unit vector tangent to $N$ at $P$. We will work out the implications of these inequalities using the notation we have already introduced but restricting our vector fields to their values at $P$.

It follows from (7) that

$$
\langle\hat{X}, \hat{X}\rangle=1-k^{2} / \lambda^{2}
$$

Further, (5) leads to the inequality $|k| \leqslant\|\operatorname{grad} \Phi\|<\varepsilon^{\prime}$ and, as $\lambda \geqslant \lambda_{0}$,

$$
\|\hat{X}\|^{2}>1-\varepsilon^{2} / \lambda_{0}^{2}
$$


The argument

$$
\begin{aligned}
\left\langle\nabla_{T} \tilde{X}, X\right\rangle & =\left\langle\nabla_{T} \hat{X}, \hat{X}\right\rangle+k^{2} / \lambda^{2} \\
& =\left\langle\nabla_{T} \hat{X}, \hat{X}\right\rangle+1-\|\hat{X}\|^{2} \\
& \geqslant 1+(\mu(a \lambda)-1)\|\hat{X}\|^{2} \\
& \geqslant 1+(\mu(a \lambda)-1)\left(1-\varepsilon^{2} / \lambda_{0}^{2}\right)
\end{aligned}
$$

uses (8), (9), (1) and (10). It then follows from (6) that

$$
\varepsilon>1+(\mu(a \lambda)-1)\left(1-\varepsilon^{\prime 2} / \lambda_{0}^{2}\right)+\langle T, S(X, X)\rangle .
$$

Given any positive integer $m$ we can take $\varepsilon=1 / m, \varepsilon^{2}=\lambda_{0}^{2} / m$ and the above inequality implies that, at some point $P_{m}$,

$$
\langle T, S(X, X)\rangle+(1-1 / m) \mu\left(a \lambda_{m}\right)<0
$$

where $\lambda_{m}=d\left(0, P_{m}\right)$. Consequently

$$
\|S(X, X)\|>(1-1 / m) \mu\left(a \lambda_{m}\right) / \lambda_{m},
$$

an inequality which we can also express as

$$
\|S(X, X)\| /\langle X, X\rangle>(1-1 / m) \mu\left(a \lambda_{m}\right) / \lambda_{m}
$$

for all non-zero vectors $X$ tangent to $N$ at $P_{m}$.

The inequality (11) shows that Lemma 2 is applicable to the function $S$. Thus, using (3) and (4), there are non-zero vectors $X$ and $Y$ tangent to $N$ at $P_{m}$ such that

$$
K_{N}(X \wedge Y)-K_{m}(X \wedge Y)=\langle S(X, X), S(Y, Y)\rangle>/\|X \wedge Y\|^{2} .
$$

Because $\|X \wedge Y\|^{2} \leqslant\|X\|^{2}\|Y\|^{2}$ the inequality (11) gives

$$
K_{N}(X \wedge Y)-K_{M}(X \wedge Y)>\left(1-\frac{1}{m}\right)^{2} \frac{\mu^{2}\left(a \lambda_{m}\right)}{\lambda_{m}^{2}} \geqslant\left(1-\frac{1}{m}\right)^{2} \frac{\mu^{2}(a \rho)}{\rho^{2}} .
$$

The fact that this is true for all $m$ proves the theorem.

We note that the inequality (*) is sharp in the sense that if $M$ is a complete simply connected space of constant sectional curvature $-a^{2}, a \geqslant 0$ and $N$ is the boundary of a closed ball of radius $\rho$, then we obtain the equality $\sup \left(K_{N}(\sigma)-K_{M}(\sigma)\right)=\mu^{2}(a \rho) / \rho^{2}$. In fact, we have $S(X, X)=\left(\mu(a \rho) / \rho^{2}\right) T$. So from (3) and (4) we obtain the above equality.

\section{References}

[1] C. Baikoussis and T. Koufogiorgos, 'Isometric immersions of complete Riemannian manifolds into euclidean space', Proc. Amer. Math. Soc. 79 (1980), 87-88.

[2] J. Cheeger and D. G. Ebin, Comparison theorems in Riemannian geometry (North-Holland, Amsterdam and Oxford, 1975).

[3] T. Ishihara, 'Radii of immersed manifolds and nonexistence of immersions', Proc. Amer. Math. Soc. 78 (1980), 276-279. 
[4] H. Jacobowitz, 'Isometric embedding of a compact Riemannian manifold into euclidean space', Proc. Amer. Math. Soc. 40 (1973), 245-246.

[5] S. Kobayashi and N. Nomizu, Foundations of differential geometry, Vol. II (Interscience, New York, 1969).

[6] J. D. Moore, 'An application of second variation to submanifold theory', Duke Math. J. 42 (1975), 191-193.

[7] H. Omori, 'Isometric immersions of Riemannian manifolds', J. Math. Soc. Japan 19 (1967), 205-214.

Department of Mathematics

University of Ioannina

Ioannina

Greece
Department of Mathematics

University of Southampton

Southampton

England 